EESTI NSV TEADUSTE AKADEEMIA TOIMETISED. XII KÖIDE FOOSIKA-MATEMAATIKA. JA TEHNIKATEADUSTE SEERIA. 1963, NR. 4

ИЗВЕСТИЯ АКАДЕМИИ НАУК ЭСТОНСКОИ ССР. ТОМ ХІІ СЕРИЯ ФИЗИКО-МАТЕМАТИЧЕСКИХ И ТЕХНИЧЕСКИХ НАУК. 1963, № 4

\title{
ОБ ИЗМЕРЕНИИ ДВОЙНОГО ЛУЧЕПРЕЛОМЛЕНИЯ ПОТОКА ОБОБЩЕННЫМ МЕТОДОМ СЕНАРМОНА
}

\author{
X. АБЕН, \\ кандидат технических наук
}

Предлагается метод измерения двойного лучепреломления потока в случае, когда компенсацнонная пластинка не является пластинкой в четверть волны.

Наряду с компенсатором Брейса $[1-4]$ и фотоэлектрическими методами $\left[{ }^{5-7}\right]$ ири измерении двойного лучепреломления потока часто применяется метод Сенармона $\left[{ }^{3,8-15}\right]$. Так как на практике разность фаз компенсационной пластинки часто отличается от $\frac{\pi}{2}$, то представляет интерес разработать метод измерения разности фаз для этого случая. Джеррард $\left.{ }^{11}\right]$ исследовал случай, когда разность фаз компенсационной пластинки мало отличается от $\frac{\pi}{2}$. В настоящей статье рассматривается измерение двойного лучепреломления потока обобщенным методом Сенармона $\left[{ }^{16}\right]$ при произвольной разности фаз компенсационной пластинки.

Рассмотрим измерение двойного лучепреломления потока в ротационном вискозиметре, когда весь зазор между цилиндрами находится в поле зрения полярископа. Именно относительно такого вискозиметра детально исследовалось применение метода Сенармона $[8,12,15]$. Оптическая схема установки представляет собой систему из двух двупреломляющих пластинок, помещенных между поляризатором и анализатором. Первой пластинкой неизвестной разностью фаз $\Delta_{1}$ является слой двупреломляющего потока, второй пластинкой - компенсационная пластинка разностью фаз $\Delta_{2}$, отличающейся в общем от $\frac{\pi}{2}$. Одно главное направление компенсационной пластинки параллельно поляризатору; анализатор можно поворачивать относительно оптической оси системы.

Если поляризатор и анализатор скрещены, то в круговом поле зрения возникают темные полосы $Q_{1}$ и $Q_{2}$ (крест изоклин), находящиеся друг от друга на угол ${ }_{2}{ }_{2}$ (рис. 1 ). В этих местах ${ }^{1}$ главные направления двупреломляющего потока параллельны и перпендикулярны поляризатору. Угол $\gamma$ называется углом угасания.

При поворачивании анализатора темные полосы перемещаются в направлении биссектрисы угла между ними, причем они одновременно просветляются $\left[{ }^{12},{ }^{15}\right]$. Если компенсационная пластинка является пластинкой

1 Из четырех темных полос на рис. 1 показаны только две. 
точно в четверть волны $\left(\Delta_{2}=\frac{\pi}{2}\right)$, то при некотором положении анализатора $\left(\alpha_{\xi}\right)$ полосы сливаются при $\beta=\frac{\pi}{4}$ (рис. 1$)$, причем в этом месте

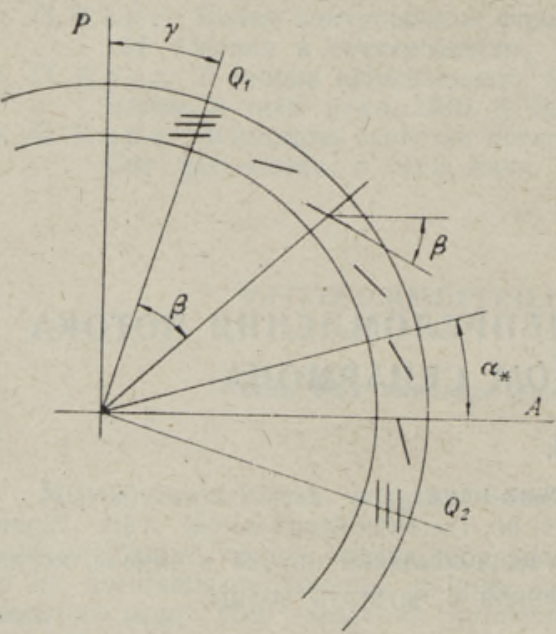

Pнс. 1. получается полное погашение света. Разность фаз $\Delta_{1}$ определяется известной формулой метода Сенармона

$$
\Delta_{1}=-2 \alpha_{*}
$$

Для исследования случая $\Delta_{2} \neq \frac{\pi}{2}$ используем метод характеристических направлений. В статье [17] было доказано, что при просвечивании стопы двупреломляющих пластинок всегда существуют два взаимно перпендикулярных направления поляризатора, при которых выходяший из стопы свет поляризован линейно Соответствующие направления колебаний выходящего света также взаимно перпендикулярны. Отмеченные направления колебаний входя-

щего света называются первичными характеристическими направлениями и выходящего света - вторичными характеристическими направлениями данной оптической системы.

В случае двух двупреломляющих пластинок характеристические направления определяются соотношениями $\left[{ }^{16-18}\right]$

$$
\begin{aligned}
& \operatorname{tg} 2 \alpha_{0}=\frac{\sin 2 \beta \sin \Delta_{2}}{\sin \Delta_{1} \cos \Delta_{2}+\cos 2 \beta \cos \Delta_{1} \sin \Delta_{2}}, \\
& \operatorname{tg} 2 \alpha_{*}=\frac{-\sin 2 \beta \sin \Delta_{1}}{\cos \Delta_{1} \sin \Delta_{2}+\cos 2 \beta \sin \Delta_{1} \cos \Delta_{2}},
\end{aligned}
$$

где $\beta$ - угол между главными направлениями пластинок, $\alpha_{0}-$ угол между главными направлениями первой пластинки и первичными характеристическими направлениями, $\alpha_{*}$ - угол между главными направлениями второй пластинки и вторичными характеристическими направлениями.

Так как главные направления компенсационной пластинки параллельны и перпендикулярны поляризатору, то угол $\beta$ (рис. 1) является углом между главными направлениями двупреломляющего потока и компенсационной пластинки. Следовательно, при рассматриваемой установке в поле зрения полярископа находится множество систем из двух двупреломляющих пластинок с разностями фаз $\Delta_{1}$ и $\Delta_{2}$ и с различными углами $\beta$, между их главными направлениями, причем угол $\beta$ изменяется от $0\left(Q_{1}\right)$, до $\frac{\pi}{2}\left(Q_{2}\right)$.

Как уже отмечалось, при $\Delta_{2}=\frac{\pi}{2}$ можно найти такое положение анализатора $\left(\alpha_{*}\right)$, при котором в месте, где $\beta=\frac{\pi}{4}$, получается полное погашение света. Исследуем, при каких условиях и при каком значении $\beta$ 
можно получить полное погашение света, если $\Delta_{2}=\frac{\pi}{2}$, и каким образом можно в этом случае определить величину $\Delta_{1}$.

Анализатор погасает полностью выходящий из второй пластинки свет только в случае, если свет поляризован линейно. Это имеет место, когда поляризатор параллелен одному из первичных характеристических направлений, т. е. $\alpha_{0}=\beta$. При $\alpha_{0}=\beta$ получим из выражения (2) следующие условия полного погашения света:

$$
\begin{gathered}
\sin 2 \beta=0, \\
\cos 2 \beta=\operatorname{ctg} \frac{\Delta_{1}}{2} \operatorname{ctg} \Delta_{2} .
\end{gathered}
$$

При $\sin 2 \beta=0$ имеем из выражения (3) $\alpha_{*}=0$. Это соответствует случаю изоклины. Из соотношения (5) следует, что при некоторых условия можно получить полное погашение света еще при определенном значении угла $\beta$. Если $\Delta_{2}=\frac{\pi}{2}$, то по соотношению (5) $\beta=\frac{\pi}{4}$ и из выражения (3) имеем

$$
\operatorname{tg} 2 \alpha_{*}=-\operatorname{tg} \Delta_{1}
$$

что соответствует описанному выше классическому методу Сенармона

Если же $\Delta_{2} \neq t \frac{\pi}{2}$, то погашение света получается не при $\beta=\frac{\pi}{4}$, а при значении $\beta$, определенном по соотношению (5). Из последнего соотношения величина $\Delta_{1}$ выражается формулой

$$
\operatorname{ctg} \frac{\Delta_{1}}{2}=\cos 2 \beta \operatorname{tg} \Delta_{2}
$$

и из соотношения (3) формулой

$$
\lg \Delta_{1}=\frac{-\sin \Delta_{2}}{\cos 2 \beta \cos \Delta_{2}+\sin 2 \beta \operatorname{ctg} 2 \alpha_{*}} .
$$

Из соотношений (7) и (8) следует возможность измерения разности фаз при $\Delta_{2} \neq \frac{\pi}{2}$. Для этого необходимо поворачивать анализатор до полного погашения света в некотором месте между $Q_{1}$ и $Q_{2}$. По известному значению $\Delta_{2}$ и по экспериментально определенному углу $\alpha_{*}$ можно из системы уравнений (7) и (8) определить $\Delta_{1}$ и $\beta$.

Еще проще определить значение $\Delta_{1}$ при помощи сетки Вульфа $\left[{ }^{19-21}\right]$ (рис. 2), которую рассматриваем как стереографическую проекцию сферы Пуанкаре. Пусть полюсы сферы Пуанкаре изображаются центром сетки Вульфа и экватор - внешним кругом. Центр сетки изображает свет, поляризованный по кругу, а внешний круг - линейно поляризованный свет. Меридианы изображают световые эллипсы, которые характеризуются относительно направлений $u$ и $v$ одинаковой разностью фаз; на параллелях отношение амплитуд колебаний $u$ и $v$ постоянно. Следует отметить, что описанная интерпретация сетки Вульфа отличается от принятого другими авторами $\left[{ }^{19,20}\right]$.

Накладываем на сетку Вульфа кальку и нарисуем меридиан $x_{2} B y_{2}$. соответствующий разности фаз $\Delta_{2}$ (рис. 3 ). Положение поляризатора Р совпадает с главным направлением второй пластинки $x_{2}$. Отметим на внешнем круге сетки положение анализатора $A$ при полном погашении света в некоторой точке между $Q_{1}$ и $Q_{2}$. Выходящий из второй пластин- 


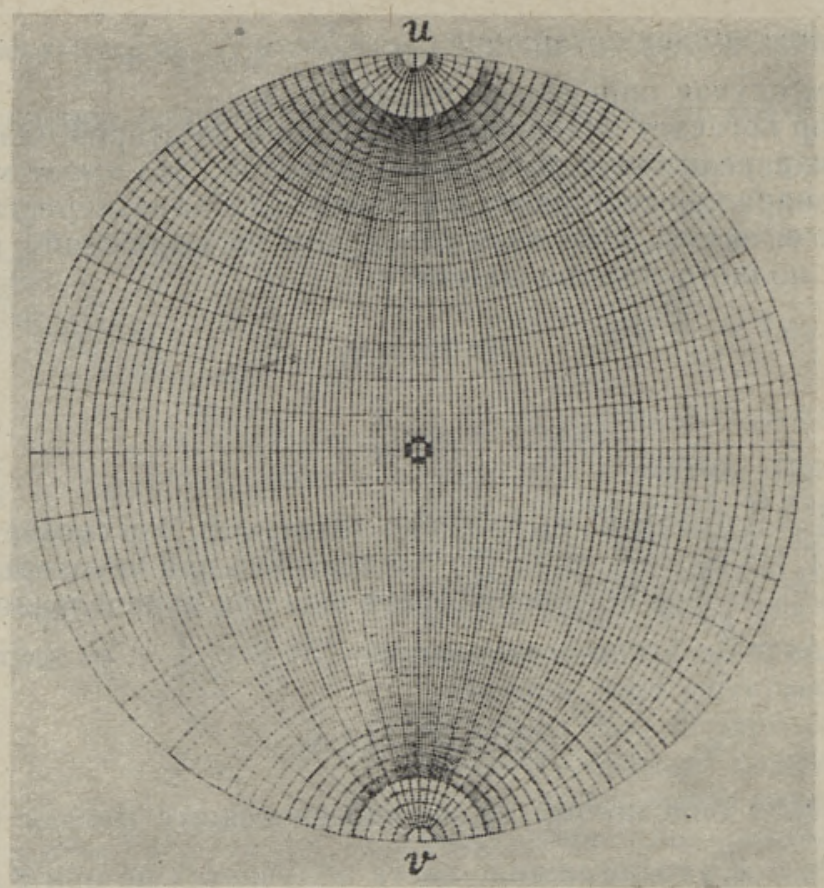

Рис. 2.

ки линейно поляризованный свет изображается точкой $A^{\prime}$. Совмещая главные направления второй пластинки $x_{2} y_{2}$ с осью сетки Вульфа $u v$,

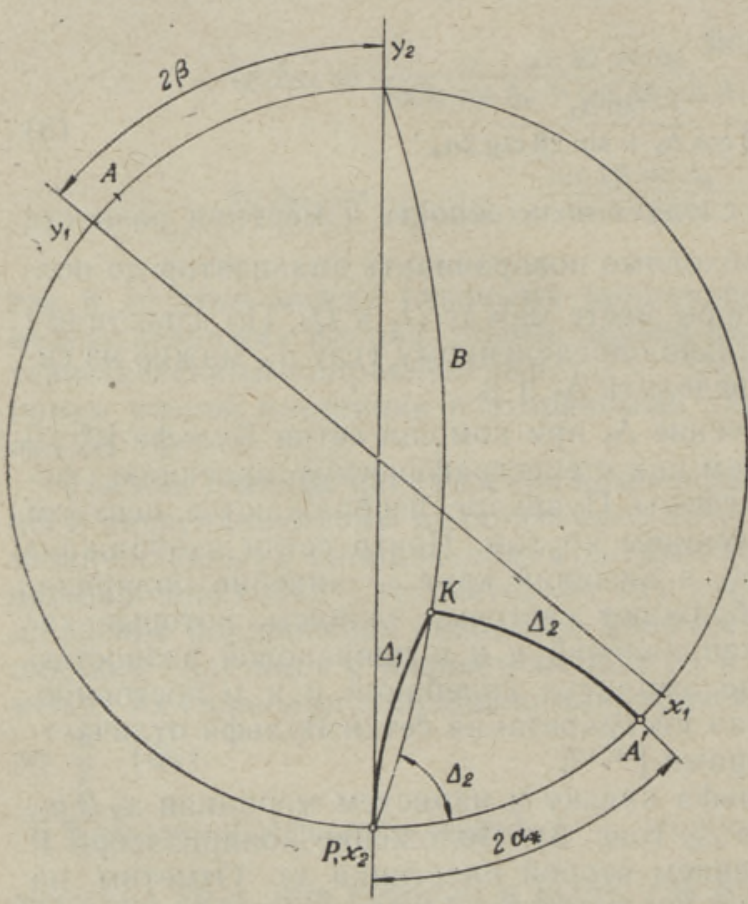

Рнс. 3. находим точку пересечения меридиана $x_{2} B y_{2}$ и параллели, проходящие через $A^{\prime}$ (точка $K$ на рис. 3). Поворачиваем кальку, пока точки $P$ и $K$ не попадут на одну параллель. Тогда ось $u v$ совпадает с главными направлениями $x_{1} y_{1}$ первой пластинки (двупреломляющего потока) и дуга $P K$ по параллели равна $\Delta_{1}$.

Пример, приведенный на рис. 3 , соответствует случаю $\Delta_{2}=70^{\circ}, \alpha_{*}=23^{\circ}$. Графическое решение дает $\Delta_{1}=60^{\circ}, \quad \beta=-25,5^{\circ}$, решая систему (7), (8) методом итераций, получим $\Delta_{1}=60,2^{\circ}, \quad \beta=$ $-25,6^{\circ}$.

Описанный метод весьма прост, и определение величины $\Delta_{1}$ требует только около минуты времени. 
Если же экспериментально определяется также угол $\beta$, то $\Delta_{1}$ можно непосредственно определить из соотношения (7) или (8).

Из соотношения (5) следует, что полное погашение света можно получить лишь тогда, когда выполнено условие

$$
\left|\operatorname{ctg} \frac{\Delta_{1}}{2}\right| \leqslant\left|\operatorname{tg} \Delta_{2}\right|
$$

так как только в этом случае угол $\beta$ имеет действительные значения. Следовательно, область измерения величины $\Delta_{1}$ ограничена. Условие (9) можно при $\Delta_{1} \leqslant 2 \pi$ переписать в виде

$$
\left|\pi-\Delta_{1}\right| \leqslant\left|2 \Delta_{2}\right| .
$$

На рис. 4 представлена область измерения $\Delta_{1}$ в зависимости от значения $\Delta_{2}$.

Интересно отметить, что область измерения $\Delta_{1}$ при заданном $\Delta_{2}$ можно расширить. Это проще всего показать на сетке Вульфа. Легко убедиться, что если поставить поляризатор не параллельно главным направлениям компенсационной пластинки, а под некоторым углом $\varphi$ относительно последних (рис. 5), то область из-

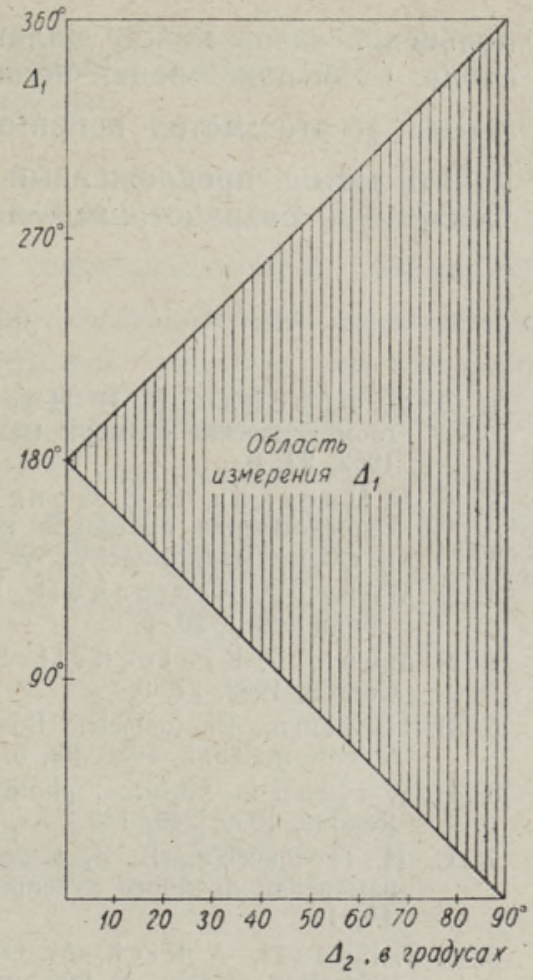

Рис. 4.

мерения $\Delta_{1}$ расширяется. От величины угла $\varphi$ зависит область измерения $\Delta_{\mathrm{l}}$, а также точность метода при различных значениях $\Delta_{1}$. Зная приближенно значение $\Delta_{1}$, можно выбрать соответствующий угол $\varphi$, дающий в этой области наивысшую точность.

Например, если при $\Delta_{2}=70^{\circ}$ область измерения величины $\Delta_{1}$ в случае $x_{2} \| P$ $(\varphi=0)$

$$
40^{\circ} \leqslant \Delta_{1} \leqslant 320^{\circ} \text {, }
$$

то при $\varphi=5^{\circ}$ можно $\Delta_{1}$ измерить в области

$$
23^{\circ} \leqslant \Delta_{1} \leqslant 337^{\circ}
$$

и при $\varphi=1^{\circ}$ в области

$$
6^{\circ} \leqslant \Delta_{1} \leqslant 354^{\circ}
$$

При этом методика измерения $\Delta_{1}$ остается прежней.

Мы рассматривали измерение разности фаз в случае применения ротацион-

Pис. 5. ного вискозиметра, при котором весь

2 ENSV TA Toimetised T-4 63 
кольцевой зазор между цилиндрами находится в поле зрения полярископа. Если поле зрения полярископа ограничено небольшим участком зазора, то этот метод неприменим, и в случае $\Delta_{2} \neq \frac{\pi}{2}$ необходимоприменять метод, предложенный Джессопом $\left[{ }^{22}\right]$, или обобщенный метод Сенармона, разработанный автором [16].

\section{Л ИТ Р РА Т Р А}

1. Э. Ф. Ф ри см ан, В. Н. Цв е тков, Динамическое двойное лучепреломление и геометрические размеры макромолекул в растворе. Ж. эксперим. и теор. физ., $1952,23,6$.

2. В. Н. Цветков, Э. В. Фри им ан, Л. С. Мухин а, Динамическое двойное лучепреломление растворов- полиметилметакрилата в различных растворителях и форма макромолекул. Ж. эксперим. и теор. физ., 1956, 30, 4.

3. P. Munk, P. Krat ochvil, Streaming birefringence, I. Collect. Czechosl. Chem. Comm., 1961, 26, 6.

4. P. Munk, P. Kratochvil, Streaming birefringence. II. Collect. Czechosl, Chem. Comm., 1962, 27, 9.

5. B. H. $\mathrm{Z}$ i m m, Photoelectric flow birefringence instrument of high sensitivity. Rev. Scient. Instrum., 1958, 29, 5.

6. H. W a y 1 a nd, Mesures photoélectriques en biréfringence d'écoulement. Comptes Rendus, 1959, 249, 14.

7. С. Н. Пеньков, В. 3. С теп аненко, Фотоэлектрическая компенсация при измерении двойного лучепреломления в потоке. Оптика и спектроскопия, 1963, 14,1 .

8. J. T. Edsall, A. Rich, M. Goldstein, An instrument for the study of double refraction of flow at low and intermediate velocity gradients. Rev. Scient. Instrum., 1952, 23, 12.

9. R. Cerf, H. A. Scheraga,' Flow birefringence in solutions of macromolecules. Chem. Rev., 1952, 51, 2.

10. A. G. Og sto n, J. E. St a n ier, A Couette viscosimeter. Biochem. J., 1953, 53, 1.

11. H. G. Jerrard, A quarter-wave compensator with a sensitive half-shadow device. J. Opt. Soc. Amer., 1954, 44, 4.

12. A. Rich, Use of the Sénarmont compensator for measuring double refraction of flow. J. Opt. Soc. Amer., 1955, 45, 5.

13. H. G. J e r r a r d, Determination of extinction directions in a doubly-refracting medium of small phase difference: Application to flow birefringence studies. J. Opt. Soc. Amer., 1956, 46, 4.

14. H. Janeschitz-Krieg l, New apparatus for measuring flow birefringence. Rev. Scient. Instrum., 1961, 31, 2.

15. C. A. Hollingworth, W. T. Granquist, Theory of measurement of flow birefringence by use of the Sénarmont compensator. J. Opt. Soc. Amer., 1962, 52, 5.

16. Х. К. А бен. Об одном подходе к измерению разности фаз при помощи фазовых пластинок. Оптика и спектроскопия, 1963, 14, 2.

17. Х. А бен, Об оптических явлениях при просвечивании стопы двупреломляющих пластинок. Изв. АН ЭССР. Сер. физ.-матем. и техн. наук, 1962, $11,1$.

18. Х. К. Абен, К теории составной пластинки в четверть волны. Оптика и спектроскопия, 1962, 13, 5 .

19. М. Ф. Бокш тей н, Графическнй расчет поляризационных преобразований. Ж. техн. физики, 1948, 18, 5.

20. М. Ф. Бокш те й н, Геометрический анализ поляризации света при просвечивании моделей из оптически чувствительных материалов. Проблемы прочности в машиностроении, 1962, вып. 8.

21. Х. К. Абен, Некоторые задачи суперпозиции двух двупреломляющих пластинок. Оптика и.спектроскопия, $1963,15,5$.

22. H. T. Jessop, On the Tardy and Sénarmont methods of measuring fractional relative retardations. Brit. J. Appl. Phys., 1953, 4, 5. 


\section{- VOOLU KAKSIKMURDVUSE MÕTTMISEST ULDISTATUD SENARMONT' MEETODIL}

H. Aben,

tehnikateaduste kandidaat

$\frac{\pi}{2}$,

Voolu kaksikmurdvuse mōōtmiseks, juhul kui kompensatsiooniplaadi faasinihe pole The riale $\left[{ }^{16},{ }^{17}\right]$. Meetod on kasutatav polariskoobi puhul, mille vaateväljas asub kogu rotatsiooniviskosimeetri pilu. Voolus tekkiva faasinihke $\Delta_{1}$ mōōtmiseks tuleb analüsaatorit pöörata kuni valguse kustumiseni mingis vaatevälja punktis. Erinevalt klassikalisest Sénarmont' meetodist ei toimu see asimuudil $\beta=\frac{\pi}{4}$ (joon. 1), vaid mingil $\beta$ väärtusel, mis on määratud seosega (5). Otsitav faasinihe $\Delta_{1}$ ja nurk $\beta$ leitakse analüsaatori pöördenurga $\boldsymbol{a}_{*}$ ja kompensatsiooniplaadi faasinihke $\Delta_{2}$ kaudu vōrrandisüsteemist (7), (8) vōi graafiliselt (joon. 3); kasutades Wulffi vôrku (joon. 2). Faasinihke $\Delta_{1}$ mõõtmispiirkond oleneb $\Delta_{2}$ väärtusest (joon. 4), kusjuures polarisaatori asendi muutmisega on mõôtmispiirkonda võimalik laiendada.

\section{Eesti NSV Teaduste Akadeemia Küberneetika Instituut \\ Saabus toimetusse \\ 28. IV 1963}

\section{ON THE MEASUREMENT OF FLOW BIREFRINGENCE BY USE OF THE GENERALIZED SENARMONT METHOD}

\section{H. Aben}

\section{Summary}

For measuring the flow birefringence in the case when the compensator plate is not an exact quarter-wave plate, a generalized Sénarmont method is developed, which is based on the theory of characteristic directions $\left[{ }^{16},{ }^{17}\right]$. The method is applicable if the whole annular space of the viscosimeter can be viewed in the polariscope. For measuring the retardation of flow $\Delta_{1}$ the analyzer must be rotated until extinction occurs somewhere in the field of the arrangement. As a difference from the classical Sénarmont method the extinction occurs not on the azimuth $\beta=\frac{\pi}{4}$ (fig. 1), but on the azimuth $\beta$ which is determined by the formula (5). The retardation $\Delta_{1}$ and the angle $\beta$ can be found from the system of equations (7), (8), where $\alpha_{*}$ is the angle of rotation of the analyzer and $\Delta_{2}$ is the retardation of the compensator plate. Much simpler is it to determine $\Delta_{1}$ and $\beta$ by a graphical method (fig. 3) using the Wulff net (fig. 2). The interval in which $\Delta_{1}$ can be measured is limited depending on $\Delta_{2}$ (fig. 4), but by rotating the polarizer this interval can be widened.

Academy of Sciences of the Estonian S.S.R., Institute of Cybernetics

Received

April 28th, 1963 